\title{
Saturation mechanism of the Weibel instability in weakly magnetized plasmas
}

\author{
Tsunehiko N. Katd* \\ National Astronomical Observatory of Japan, \\ 2-21-1 Osawa, Mitaka, Tokyo 181-8588, Japan
}

(Dated: 27 June, 2005)

\begin{abstract}
The saturation mechanism of the Weibel instability is investigated theoretically by considering the evolution of currents in numerous cylindrical beams that are generated in the initial stage of the instability. Based on a physical model of the beams, it is shown that the magnetic field strength attains a maximum value when the currents in the beams evolve into the Alfvén current and that there exist two saturation regimes. The theoretical prediction of the magnetic field strength at saturation is in good agreement with the results of two-dimensional particle-in-cell simulations for a wide range of initial anisotropy.
\end{abstract}

*Electronic address: katoutn@cc.nao.ac.jp 
Collisionless plasmas with anisotropic velocity distributions drive the Weibel instability 1, 2], thereby generating magnetic fields. Recently, this instability in astrophysical as well as laboratory plasmas has attracted considerable attention. For example, it is considered that this instability can be driven in strong collisionless shock waves associated with various astrophysical phenomena, e.g., pulsar winds [3, 4], gamma-ray bursts and/or their afterglows [5], or gravitational collapse of large-scale structures in the universe [6]. It can also be driven if temperature gradients are present in plasmas [7]. The magnetic field generated by the instability is responsible for synchrotron and/or "jitter" radiation from the existing highenergy particles [8]. Furthermore, such magnetic fields can provide an effective scattering mechanism for charged particles. For example, it would affect the dissipation process of collisionless shock waves, efficiency of the Fermi acceleration, or heat conductivity by charged particles. In all these cases, the amplitude of the magnetic field is of primary importance.

The magnetic field strength attains the maximum value at the saturation of the instability. Several conditions for saturation have been proposed [5, 9, 10]. However, these conditions may be unsatisfactory because the role of current was not treated explicitly in these studies. Since the instability enters the nonlinear regime before saturation, the currents and fields must be considered self-consistently. As shown by numerical simulations, the currents are carried by numerous cylindrical beams [11, 12, 13]. Therefore, the physics of saturation will be elucidated by examining the evolution of such beams.

In this letter, the nonlinear saturation mechanism of the Weibel instability, i.e., that of the transverse modes of filamentation instability, is investigated with a physical model of the beams. The theoretical analysis and simulations are performed within a two-dimensional framework. (Therefore, in three dimensions, the results might be modified by other effects.) The method is applicable to electron-proton as well as electron-positron plasmas. However, for electron-proton plasmas, only the saturation due to the electron currents is considered. Although proton currents later cause the second saturation [13], it is beyond the scope of the present study and will be investigated in the future. In the following analysis, protons are treated as a charge neutralizing background.

Theoretical model of saturation - In this section, the saturation mechanism is modeled by using Gaussian units and the following notation: speed of light, $c$; velocity of a particle normalized by the speed of light, $\boldsymbol{\beta}$; Lorentz factor of the particle, $\gamma \equiv\left(1-\boldsymbol{\beta}^{2}\right)^{-1 / 2}$; electron mass, $m_{e}$; electron charge, $-e$; proton mass, $m_{p}$; mean electron number density, 
$n_{e}$; electron plasma frequency, $\omega_{p e} \equiv\left(4 \pi n_{e} e^{2} / m_{e}\right)^{1 / 2} ;$ electron skin depth, $l_{0} \equiv c / \omega_{p e} \approx$ $5.3 \times 10^{5} n_{e}^{-1 / 2}[\mathrm{~cm}]$.

The initial condition is set as follows. The plasma is initially uniform and unmagnetized (or weakly magnetized) with an anisotropic velocity distribution. The initial velocity distributions of the particles are axisymmetric, and the velocity dispersion along the $z$-axis is larger than those along the other axes. For electron-positron plasmas, the distribution functions are the same for both species. For electron-proton plasmas, the distribution function of only the electrons is considered. Such a distribution function would be plausible for two-stream like conditions [4, 5, 6, 11, 12, 13, 14].

The basic physical mechanism of the instability is described as follows. In the initial stage of the instability, the charged particles in the plasma are deflected by small magnetic fluctuations. In the present condition, most particles have larger velocities along the $z$ axis. Since the particles carrying current toward the $+z$ direction and $-z$ direction are deflected in opposite directions, they are separated into different regions, thereby producing net currents in the plasma that generate magnetic fields [5]. As the amplification of the magnetic fields increases, the distance between the two populations of particles increases, and the currents are amplified, and vice-versa. Thus, both currents and magnetic fields increase exponentially. In this evolution, it is important for the instability to enter the nonlinear regime before saturation. As revealed by two- or three-dimensional simulations 11, 12, 13], many isolated cylindrical beams (or current filaments) are formed in the plasma long before saturation; each beam carries a net current and generates a magnetic field around itself. Since two beams with currents in the same direction attract each other and tend to coalesce into a larger beam, the beams grow with time [11, 12] even before saturation.

Based on these observations, we model the nonlinear evolution of the instability from the "initial condition" in which the plasma consists of many cylindrical beams to saturation. Initially, the radius and magnitude of current in the beams are almost uniform because the plasma is homogeneous. Each beam carries a net current in either the $+z$ or $-z$ direction. Subsequently, both the current and magnetic field in each beam increase exponentially due to particle separation in the beam. The radii of the beams also increase due to the coalescing process, although it is considerably slower than the increase in current and magnetic field. However, they are still considered to be uniform because they essentially evolve equally. Therefore, the plasma can be modeled as an ensemble of uniform cylindrical beams with 
the same radius $r$ and magnitude of current $I$. (A similar model was recently considered in Ref. [15] for investigating the evolution after saturation.) By assuming a uniform current density $J$, where $I=\pi r^{2} J$, the root-mean-square value of the magnetic field strength within a cylindrical beam is calculated using the Ampère's law as follows:

$$
B=\sqrt{2} \pi r J / c=\sqrt{2} I /(r c)
$$

Although in practice, the magnetic field is affected by neighboring beams, their net effect will be low and the order of the magnetic field strength in the beam will be given by Eq. (II). Thus, we assume that the magnetic field (11) in each beam is essential to the saturation process and those generated by the neighboring beams are important only in the coalescing process.

On the basis of this model, we consider the evolution of one of the beams. The exponential increase in current and magnetic field ceases when the magnitude of the current is equal to the smaller of the following two upper limits. One limit is the Alfvén current [16], which occurs due to the self-generated magnetic field; it is expressed in Gaussian units by

$$
I_{A}=I_{0}\left\langle\gamma \beta_{\|}\right\rangle
$$

where $I_{0} \equiv m_{e} c^{3} / e(\approx 17000[\mathrm{~A}]), \beta_{\|}$is the magnitude of the $z$ component of $\boldsymbol{\beta}$, and the angle brackets \langle\rangle denote an average taken over the beam volume. (This limit was originally derived for a monoenergetic single-directed particle beam. In this case, the averaged value of $\gamma \beta_{\|}$is used instead of that in the monoenergetic cases because the energy of particles is distributed.) We can apply this limit even when the separation process is in progress and a fraction of particles carry current in the opposite direction because the self-generated magnetic field is associated with the net current $I$, and it always ensures that $I$ does not exceed $I_{A}$. The other limit is the maximum current $I_{P}$ that can be carried by all the particles within the beam:

$$
I_{P}=\pi r^{2} J_{P}, \quad \text { where } \quad J_{P} \equiv e \mu n_{e}\left\langle\beta_{\|}\right\rangle c
$$

with $\mu=1$ for electron-proton plasmas and $\mu=2$ for electron-positron plasmas. This limit is reached when the separation of the current density is completed before $I$ equals $I_{A}$. Since the distribution of particles in the beam approaches an isotropic distribution depending on the evolution of the instability, we must consider this effect; it is expressed by a factor $\chi$ as 
follows:

$$
\left\langle\beta_{\|}\right\rangle=\chi\left\langle\beta_{\|}\right\rangle_{0}, \quad\left\langle\gamma \beta_{\|}\right\rangle=\chi\left\langle\gamma \beta_{\|}\right\rangle_{0}
$$

where subscript "0" denotes an initial value. Since a complete isotropization of the strong initial anisotropy yields the lower limit $\chi=1 / 3$, we observe that $1 / 3<\chi<1$.

After the exponential growth ceases, the beam evolves through the coalescing process. In the former case $\left(I_{A}<I_{P}\right)$, which is termed the Alfvén limit, once the current equals the Alfvén current $I_{A}$, it no longer increases and it retains the value of $I_{A}$, while the radius continues to increase. Therefore, from Eq. (11), the magnetic field decreases monotonically thereafter. In the latter case $\left(I_{A}>I_{P}\right)$, which is termed the particle limit, current gradually increases until it reaches the Alfvén current; during this period, it increases with the radius while satisfying the condition $I=\pi r^{2} J_{P}$, and the magnetic field also increases. After the current equals the Alfvén current, the magnetic field decreases monotonically, as in the Alfvén limit.

Therefore, for both limits, the magnetic field strength becomes maximum when the current evolves into the Alfvén current. From Eq. (11), the maximum value $B_{\max }$ is expressed as follows:

$$
B_{\max }=\sqrt{2} I_{A} /(\tilde{r} c)=\sqrt{2}\left\langle\gamma \beta_{\|}\right\rangle\left(\tilde{r} / l_{0}\right)^{-1} B_{*}
$$

where $\tilde{r}$ is the beam radius at saturation and $B_{*}$ is the magnetic field strength given by $B_{*} \equiv c\left(4 \pi n_{e} m_{e}\right)^{1 / 2} \approx 3.2 \times 10^{-3} n_{e}^{1 / 2}[\mathrm{G}]$. In the Alfvén limit, the radius at saturation $\tilde{r}_{A}$ is related to the initial radius $r_{0}$ or the wavelength of the most unstable mode in the linear theory $\lambda_{0}$. Here, it is simply expressed using two factors $\alpha$ and $\zeta$ as follows:

$$
\tilde{r}_{A}=\alpha r_{0}=\alpha \zeta \lambda_{0}
$$

Thus, the magnetic field at saturation is given by

$$
B_{\max }=\sqrt{2} c_{A}\left\langle\gamma \beta_{\|}\right\rangle_{0}\left(\lambda_{0} / l_{0}\right)^{-1} B_{*}
$$

where $c_{A} \equiv \chi_{A} /(\alpha \zeta)$, and $\chi_{A}$ denotes the effect of isotropization at saturation [see Eq. (4)]. In the particle limit, the radius at saturation $\tilde{r}_{P}$ is determined from the condition $\pi \tilde{r}_{P}^{2} J_{P}=I_{A}$ as follows:

$$
\tilde{r}_{P}=\left(I_{A} / \pi J_{P}\right)^{1 / 2}=2 l_{0}\left(\left\langle\gamma \beta_{\|}\right\rangle_{0} / \mu\left\langle\beta_{\|}\right\rangle_{0}\right)^{1 / 2}
$$


which is on the order of $l_{0}$ for nonrelativistic cases. Using this radius, the maximum magnetic field strength is given by

$$
B_{\max }=\chi_{P}\left(\mu\left\langle\gamma \beta_{\|}\right\rangle_{0}\left\langle\beta_{\|}\right\rangle_{0} / 2\right)^{1 / 2} B_{*}
$$

where $\chi_{P}$ is the isotropization factor at saturation. As mentioned earlier, the values of $B_{\text {max }}$ in Eqs. (7) and (9) can be regarded as typical magnetic fields of the entire plasma at saturation in the respective cases. The two parameters $\left(c_{A}\right.$ and $\left.\chi_{P}\right)$ in these expressions are expected to be of order unity.

The ratio of the maximum magnetic energy density to the initial particle kinetic energy density, $\eta$, is written as $\eta=\left(B_{\max } / B_{*}\right)^{2}[2 \mu(\langle\gamma\rangle-1)]^{-1}$. (It should be noted that for electron-proton plasmas, the kinetic energy of protons is not considered in this equation.) For the particle limit regime, $\eta$ yields a subequipartition value. In particular, $\eta \sim \chi_{P}^{2} / 2$ for nonrelativistic plasmas and $\eta \sim \chi_{P}^{2} / 4$ for ultrarelativistic plasmas. The conclusion that $\eta$ yields a subequipartition value for strongly anisotropic cases is in agreement with that of previous simulations [4, 9, 10, 12, 14].

Comparison with numerical simulations - The results of the developed analytical model are compared with those of numerical simulations for electron-positron and electron-proton plasmas $\left(m_{p} / m_{e}=1836\right)$. The simulation code used is a relativistic, electromagnetic, particle-in-cell code with two spatial and three velocity dimensions. This code was developed based on a general description by Ref. [17]. The $x-y$ plane perpendicular to the $z$-axis is considered to be the simulation plane. The initial particle distribution is expressed in terms of the normalized momentum $\mathbf{u} \equiv \gamma \boldsymbol{\beta}$ that is common for all the species. Each component of $\mathbf{u}$ obeys the Gaussian distribution with a standard deviation of $\sigma_{\|}$for the $z$ component or $\sigma_{\perp}$ for the other components. The simulations were performed using a $512 \times 512$ grid with $\sim 50$ particles per cell per species under periodic boundary conditions for several values of $\sigma_{\|}$ranging from 0.12 to 10 with fixed $\sigma_{\perp}=0.1$. For each simulation, the physical size of the simulation box in each direction, $L$, was considered to be at least 7 times larger than the typical beam diameter at saturation, which was estimated by a preliminary simulation [e.g., $L=120 l_{0}$ for $\sigma_{\|}=0.12$ (largest); $L=30 l_{0}$ for $\sigma_{\|}=0.6$ (smallest)]. Then, in each simulation, the evolution of the magnetic field strength averaged over the simulation box was monitored and its maximum value was obtained; the calculation time $T$ considered was long enough to obtain the maximum value [e.g., $T=1500 \omega_{\mathrm{pe}}^{-1}$ for $\sigma_{\|}=0.12$ (longest); 
$T=25 \omega_{\mathrm{pe}}^{-1}$ for $\sigma_{\|}=1.1$ (shortest)]. Figure 1 shows the obtained maximum values denoted by dots as a function of the initial anisotropy, $\sigma_{\|} / \sigma_{\perp}-1$ : (a) electron-positron plasma and (b) electron-proton plasma.

The corresponding theoretical results are obtained as follows: First, in Eqs. (71) and (91), we approximate that $\left\langle\gamma \beta_{\|}\right\rangle_{0}=\sigma_{\|}$and $\left\langle\beta_{\|}\right\rangle_{0}=\sigma_{\|} / \tilde{\gamma}$, where $\tilde{\gamma} \equiv\left(1+\sigma_{\|}^{2}+2 \sigma_{\perp}^{2}\right)^{1 / 2}$. Next, in the present case, since the linear theory of nonrelativistic weak anisotropic plasmas [9] is applicable to the Alfvén limit regime, we obtain

$$
\lambda_{0}=2 \pi \sqrt{3 / \mu}\left[\left(\sigma_{\|} / \sigma_{\perp}\right)^{2}-1\right]^{-1 / 2} l_{0}
$$

(It should be noted that, when $\sigma_{\perp}>1$ or $\sigma_{\|}>1$, the relativistic dispersion relation must be employed.) Finally, using these expressions, we obtain

$$
\frac{B_{\max }}{B_{*}}=\left\{\begin{array}{r}
\sqrt{\frac{\mu}{6} \frac{c_{A} \sigma_{\|}}{\pi}\left[\left(\sigma_{\|} / \sigma_{\perp}\right)^{2}-1\right]^{1 / 2}} \quad \text { (Alfvén limit) } \\
\chi_{P} \sigma_{\|}[\mu /(2 \tilde{\gamma})]^{1 / 2} \text { (particle limit) }
\end{array}\right.
$$

The results are plotted in Fig. 1; dotted curves represent the results for the Alfvén limit and solid curves for the particle limit. The parameters $\left(c_{A}\right.$ and $\left.\chi_{P}\right)$ are assumed to be constants. Their values are taken as $\left(c_{A}, \chi_{P}\right)=(1.2,0.5)$ in $(\mathrm{a})$ and $(1.0,0.5)$ in (b) to match with the simulation results; they are of order unity, as expected. The transition anisotropy is given by $\left(\sigma_{\|} / \sigma_{\perp}\right)_{c} \approx\left[1+3 \pi^{2}\left(\chi_{P} / c_{A}\right)^{2}\right]^{1 / 2}$, and $\left(\sigma_{\|} / \sigma_{\perp}\right)_{c} \approx 2.5$ for $(\mathrm{a})$ and $\approx 2.9$ for $(\mathrm{b})$. These curves are in good agreement with the simulation results in their respective regions. It is evident that there are two saturation regimes. We also observe that the assumption of the constancy of $c_{A}$ and $\chi_{P}$ holds true over a wide range of anisotropy in both the figures, although the theoretical curves deviate slightly from those of the simulation results in the weak anisotropy side.

Other comparisons are shown below for electron-positron plasmas. Using Eq. (11), we can indirectly estimate $r$ from the simulation results:

$$
r=(2 \pi \sqrt{2})^{-1}\left(B / B_{*}\right)\left(S / l_{0}^{2}\right)\left(I_{\text {tot }} / I_{0}\right)^{-1} l_{0},
$$

where $S$ is the area of the simulation box and $I_{\text {tot }}$ is the total current in one direction along the $z$-axis. Figure 2(a) compares the radius at saturation obtained from this equation with those of the model ( $\tilde{r}_{A}$ [Eqs. (6) and (10) with $\left.\alpha \zeta=0.7\right]$ and $\tilde{r}_{P}[$ Eq. (8) $]$ ). Figure 2(b) shows the current per beam at saturation estimated using the radius of Eq. (12) normalized 
by the Alfvén current $I_{A}=I_{0} \sigma_{\|}$, which does not include the isotropization effect. Hence, the normalized values are not expected to be unity but to be close to the isotropization factor; $\chi_{A}=c_{A} \alpha \zeta=0.84$ for the Alfvén limit, and $\chi_{P}=0.5$ for the particle limit (shown by dotted lines). We observe that the model is consistent with the simulations. Figure 3 shows the current density at saturation obtained from the simulations for three cases: (a) Alfvén limit regime $\left(\sigma_{\|}=0.15, \chi=0.84\right)$, (b) particle limit regime $\left(\sigma_{\|}=3.1, \chi=0.5\right)$, and (c) the transition point $\left(\sigma_{\|}=0.3, \chi=0.5\right)$. The current density is normalized by $J_{P}$, which includes the isotropization effect. It is evident that saturation occurred when $\left|J_{z}\right| \approx J_{P}$ for (b) and (c), while $\left|J_{z}\right| \ll J_{P}$ for (a). It should be noted that in some regions of (b) and (c), the current density exceeds $J_{P}$. This occurs because the current-carrying beams are pinched. In any case, the typical magnetic field can still be estimated from Eq. (10) because it is mainly determined by the total current in a beam, which approximates to the Alfvén current at saturation irrespective of whether the beam is pinched or not.

Discussion - Even in the presence of a background magnetic field, the proposed model is applicable if the time taken for saturation is shorter than the deflection time due to the background magnetic field. Otherwise, the problem of the magnetized Weibel instability or the whistler instability for electron-proton plasmas [18] should be considered.

It is shown that the proposed model is consistent with several conditions obtained previously. Medvedev and Loeb [5] proposed that saturation occurs when the effective Larmor radius $r_{L}(B)$ becomes comparable to the most unstable wavelength in linear theory, $\lambda_{0}$. If $\lambda_{0}$ is replaced by the typical beam radius at saturation, $\tilde{r}$, we obtain the condition of $r_{L}\left(B_{\max }\right) \approx \tilde{r}$, which is qualitatively equivalent to condition (5). Califano et al. 14] also found that saturation occurs when $r_{L}(B) \approx l_{0}$. This result is in agreement with the result of the particle limit in Eq. (8)

In electron-proton plasmas, if the initial velocity distribution of the protons is the same as that of electrons, the upper limits for the proton currents will be given by $I_{A}^{\prime}=\left(m_{p} / m_{e}\right) I_{A}$ and $I_{P}^{\prime}=I_{P}$ with $\mu=1$. Thus, for the particle limit, both $\tilde{r}_{P}$ and $B_{\max }$ would be $\left(m_{p} / m_{e}\right)^{1 / 2}$ times larger than those of the electron currents.

In conclusion, the magnetic field generated by the Weibel instability saturates when the currents in the beams evolve into the Alfvén current; there are two saturation regimes: the Alfvén limit and the particle limit. The beam model proposed in this letter provides a good estimate of the magnetic field strength at saturation. This model will also be useful to 
consider the evolution of magnetic fields even after saturation.

\section{Acknowledgments}

I would like to thank M. Hattori, Y. Fujita, and N. Okabe for their helpful discussions. This research is partly supported by the Japan Science and Technology Agency. 
[1] E. S. Weibel, Phys. Rev. Lett. 2, 83 (1959).

[2] B. D. Fried, Phys. Fluids 2, 337 (1959).

[3] M. Hoshino, in Neutron stars and pulsars, edited by N. Shibazaki et al. (Univ. Academy Press, 1998), p. 491

[4] Y. Kazimura, J. I. Sakai, T. Neubert, and S. V. Bulanov, Astrophys. J. Lett. 498, L183 (1998).

[5] M. V. Medvedev and A. Loeb, Astrophys. J. 526, 697 (1999).

[6] R. Schlickeiser and P. K. Shukla, Astrophys. J. Lett. 599, L57 (2003).

[7] N. Okabe and M. Hattori, Astrophys. J. 599, 964 (2003).

[8] M. V. Medvedev, Astrophys. J. 540, 704 (2000).

[9] R. C. Davidson, D. A. Hammer, I. Haber, and C. E. Wagner, Phys. Fluids 15, 317 (1972).

[10] T.-Y. B. Yang, J. Arons, and A. B. Langdon, Phys. Plasmas 1, 3059 (1994).

[11] R. Lee and M. Lampe, Phys. Rev. Lett. 31, 1390 (1973).

[12] L. O. Silva, R. A. Fonseca, J. W. Tonge, J. M. Dawson, W. B. Mori, and M. V. Medvedev, Astrophys. J. Lett. 596, L121, (2003).

[13] J. T. Frederiksen, C. B. Hededal, T. Haugbølle, and Å. Nordlund, Astrophys. J. Lett. 608, L13, (2004).

[14] F. Califano, F. Pegoraro, S. V. Bulanov, and A. Mangeney, Phys. Rev. E 57, 7048 (1998).

[15] M. V. Medvedev, M. Fiore, R. A. Fonseca, L. O. Silva, and W. B. Mori, Astrophys. J. Lett. 618, L75, (2005).

[16] H. Alfvén, Phys. Rev. 55, 425 (1939).

[17] C. K. Birdsall and A. B. Langdon, Plasma Physics via Computer Simulation (IOP Publishing, Bristol, 1991).

[18] S. L. Ossakow, I. Haber, and E. Ott, Phys. Fluids 15, 1538 (1972). 


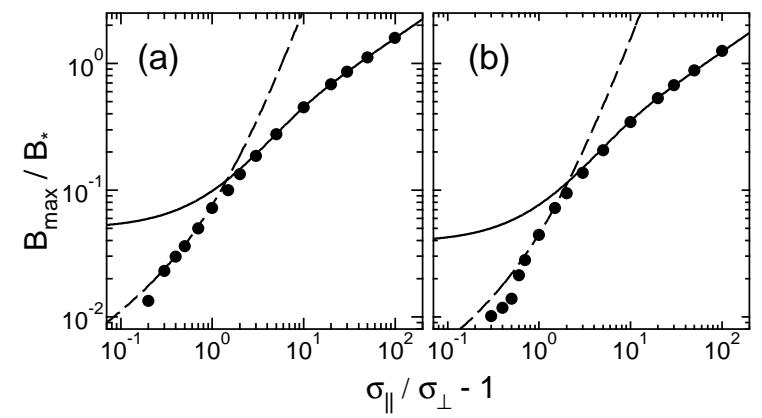

FIG. 1: Maximum magnetic field strength $B_{\max }$ for $\sigma_{\perp}=0.1$ as a function of the initial anisotropy, $\sigma_{\|} / \sigma_{\perp}-1$ : (a) electron-positron plasma, and (b) electron-proton plasma. Dots denote the results of numerical simulations. The dashed and solid curves represent the theoretical results for the Alfvén limit and particle limit, respectively [see Eq. (11)]. This shows good agreement between the predictions of the proposed theory and the simulation results in each saturation regime. 


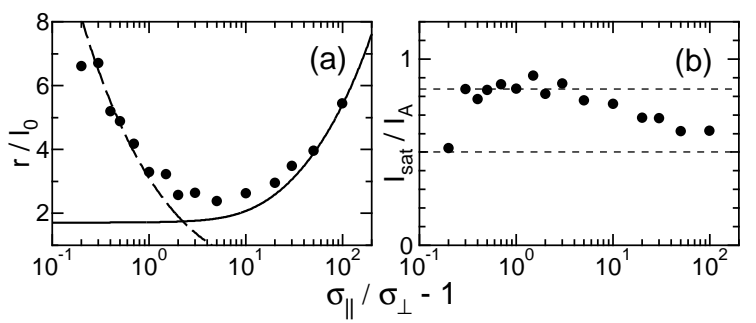

FIG. 2: (a) Typical beam radius at saturation for the electron-positron plasma as a function of the initial anisotropy with $\sigma_{\perp}=0.1$. Dots represent the radii of Eq. (12) evaluated using the simulation results. The dashed curve represents $\tilde{r}_{A}$ [see Eq. [6] ]; solid curve $1.2 \tilde{r}_{P}$ [see Eq. (8)] ]. (b) Current per beam at saturation obtained from the simulation results normalized by the Alfvén current $\left(I_{A}=I_{0} \sigma_{\|}\right)$. The expected values are represented by the dotted lines (see text). The results of the theoretical model are consistent with those of the numerical simulations. 


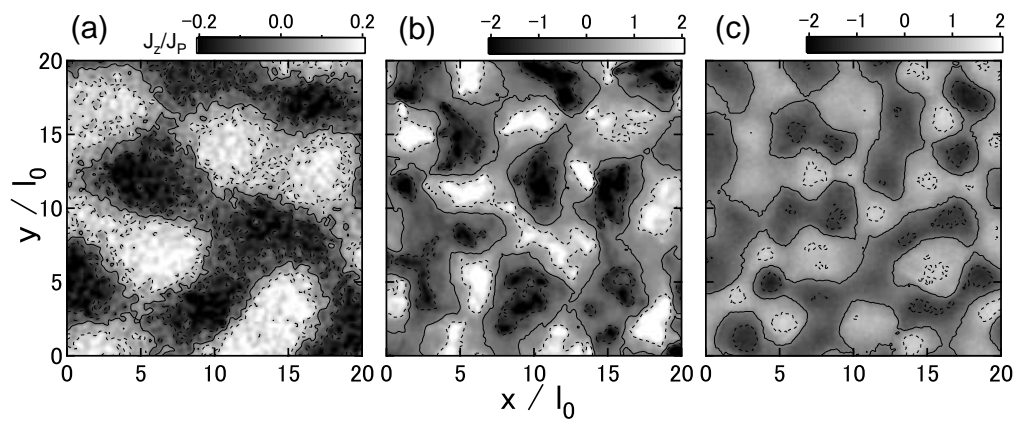

FIG. 3: Contour plots of current density at saturation, $J_{z}$, normalized by $J_{P}$, on the $x-y$ plane for $\sigma_{\perp}=0.1$ : (a) Alfvén limit regime $\left(\sigma_{\|}=0.15\right)$, (b) particle limit regime $\left(\sigma_{\|}=3.1\right.$ ), and (c) the transition anisotropy $\left(\sigma_{\|}=0.3\right)$. The spatial unit is the electron skin depth $l_{0}$. The dotted curves indicate the levels of $J_{z} / J_{P}= \pm 0.1$ in (a), and those of $J_{z} / J_{P}= \pm 1$ in (b) and (c). The solid curves indicate the levels of $J_{z}=0$ in all figures. We observe that saturation occurs when $\left|J_{z}\right|<J_{P}$ in (a) and $\left|J_{z}\right| \sim J_{P}$ in (b) and (c), as predicted by the proposed theory. This figure also confirms that the beams are approximately uniform in radius and current for all the cases, which are assumed in the theoretical model. 